\title{
Transmutations for Darboux transformed operators with applications
}

\author{
Vladislav V. Kravchenko and Sergii M. Torba \\ Department of Mathematics, CINVESTAV del IPN, Unidad Queretaro, \\ Libramiento Norponiente No. 2000, Fracc. Real de Juriquilla, Queretaro, \\ Qro. C.P. 76230 MEXICO e-mail: vkravchenko@qro.cinvestav.mx*
}

June 15, 2018

\begin{abstract}
We solve the following problem. Given a continuous complex-valued potential $q_{1}$ defined on a segment $[-a, a]$ and let $q_{2}$ be the potential of a Darboux transformed Schrödinger operator, that is $q_{2}=-q_{1}+2\left(\frac{f^{\prime}}{f}\right)^{2}$ where $f$ is a nonvanishing solution of the equation $A_{1} f=\left(\frac{d^{2}}{d x^{2}}-q_{1}(x)\right) f=0$. Suppose a transmutation operator $\mathbf{T}_{1}$ is known such that $A_{1} \mathbf{T}_{1} u=\mathbf{T}_{1} \frac{d^{2}}{d x^{2}} u$ for any $u \in C^{2}[-a, a]$. Find an analogous transmutation operator for $A_{2}=\frac{d^{2}}{d x^{2}}-q_{2}(x)$.

It is well known that the transmutation operators can be realized in the form of Volterra integral operators with continuously differentiable kernels. Given a kernel $K_{1}$ of the transmutation operator $\mathbf{T}_{1}$ we find the kernel $K_{2}$ of $\mathbf{T}_{2}$ in a closed form in terms of $K_{1}$. As a corollary interesting commutation relations between $\mathbf{T}_{1}$ and $\mathbf{T}_{2}$ are obtained which then are used in order to construct the transmutation operator for the one-dimensional Dirac system with a scalar potential.
\end{abstract}

\section{Introduction}

Transmutation operators are a widely used tool in the theory of linear differential equations (see, e.g., [2], 4], 23, 25, 35 and the recent review 33]). It is well known that under certain quite general conditions the transmutation operator transmuting the operator $A=-\frac{d^{2}}{d x^{2}}+q(x)$ into $B=-\frac{d^{2}}{d x^{2}}$ is a Volterra integral operator with good properties. Its kernel can be obtained as a solution of a certain Goursat problem for the Klein-Gordon equation with a variable coefficient. In the book 8 another approach to the transmutation was developed. It was shown that to every (regular) linear secondorder ordinary differential operator $L$ one can associate a linear space spanned on a so-called $L$-basis - an infinite family of functions $\left\{\varphi_{k}\right\}_{k=0}^{\infty}$ such that $L \varphi_{k}=0$ for $k=0,1, L \varphi_{k}=k(k-1) \varphi_{k-2}$, for $k=2,3, \ldots$ and all $\varphi_{k}$ satisfy certain prescribed initial conditions. Then the operator of transmutation was introduced as an operation transforming functions from one such linear space corresponding to a certain operator $L$ to functions from another linear space corresponding to another operator $M$, and the transformation consists in substituting the $L$-basis with the $M$-basis preserving the same coefficients in the expansion.

In [3] the relation between the transmutation operator in the form of a Volterra integral operator and as a transmutation of the bases was clarified. In particular, it was shown that the corresponding Volterra integral transmutation operator transmutes the powers of the independent variable $x^{k}$ into the elements $\varphi_{k}$ of an appropriate $L$-basis. In order to assure such an important mapping property

\footnotetext{
* Research was supported by CONACYT, Mexico. Research of second named author was supported by DFFD, Ukraine (GP/F32/030) and by SNSF, Switzerland (JRP IZ73Z0 of SCOPES 2009-2012).
} 
of the transmutation operator a parametrized family of transmutation operators for the Schrödinger operator $A$ was introduced. This parametrized family resulted to be a necessary and natural tool for solving the problem of construction of the transmutation operator for a Darboux transformed Schrödinger operator. The solution to this problem is the main aim of the present paper.

We give an explicit representation for the kernel of the transmutation operator corresponding to the Darboux transformed potential in terms of the transmutation kernel for its superpartner (Theorem 19). Moreover, this result leads to interesting commutation relations between the two transmutation operators (Corollary 25) which in their turn allow us to obtain a transmutation operator for the onedimensional Dirac system with a scalar potential as well as to prove (Theorem 26) the main property of the transmutation operator under less restrictive conditions than it has been proved until now. Namely, we show that the considered transmutation operators transmute $A$ into $B$ for any continuous (and not necessarily differentiable) potential $q$ admitting a nonvanishing, in general, complex-valued solution (and this condition is always fulfilled, e.g., when $q$ is real valued). We give several examples of explicitly constructed kernels of transmutation operators. It is worth mentioning that in the literature there are very few explicit examples and even in the case when $q$ is a constant such kernel was presented recently in [3]. The results of the present paper allow us to enlarge considerably the list of available examples and give a relatively simple tool for constructing Darboux related sequences of the transmutation kernels.

In Section 2 we introduce two main objects, the transmutation operators and the systems of recursive integrals or the $L$-bases. Besides recalling some recent results from [3] on the parametrized family of the transmutation operators we prove (Theorem 6) a criterion for a function to be a kernel of a transmutation operator from the parametrized family as well as a result on the explicit form of the inverse transmutation operator (Theorem 10). In Section 3 we present the construction of the transmutations for the Darboux transformed operators and obtain the commutation relations for them. We use the relations to weaken the conditions on the potential under which the transmutation operators preserve their main property (Theorem 26). The section ends with several examples of explicitly constructed transmutation kernels. In Section 4 the results of the preceding section are used in order to obtain the transmutation operator for the one-dimensional Dirac system with a scalar potential. Finally, Section 5 contains some concluding remarks.

\section{Transmutation operators for Sturm-Liouville equations and systems of recursive integrals}

\subsection{Transmutation operators}

According to the definition given by Levitan [23, let $E$ be a linear topological space, $A$ and $B$ be linear operators: $E \rightarrow E$. Let $E_{1}$ and $E_{2}$ be closed subspaces of $E$.

Definition 1 A linear invertible operator $T$ defined on the whole $E$ and acting from $E_{1}$ to $E_{2}$ is called a transmutation operator for the pair of operators $A$ and $B$ if it fulfills the following two conditions.

1. Both the operator $T$ and its inverse $T^{-1}$ are continuous in $E$;

2. The following operator equality is valid

$$
A T=T B
$$

or which is the same

$$
A=T B T^{-1} .
$$

Our main interest concerns the situation when $A=-\frac{d^{2}}{d x^{2}}+q(x), B=-\frac{d^{2}}{d x^{2}}$, and $q$ is a continuous complex-valued function. Hence for our purposes it will be sufficient to consider the functional space $E=C^{2}[a, b]$ with the topology of uniform convergence. For simplicity we will assume that the interval is symmetric with respect to the origin, thus $E=C^{2}[-a, a]$. 
An operator of transmutation for such $A$ and $B$ can be realized in the form (see, e.g., 23] and [25]) of a Volterra integral operator

$$
T u(x)=u(x)+\int_{-x}^{x} K(x, t) u(t) d t
$$

where $K(x, t)=H\left(\frac{x+t}{2}, \frac{x-t}{2}\right)$ and $H$ is the unique solution of the Goursat problem

$$
\begin{gathered}
\frac{\partial^{2} H(u, v)}{\partial u \partial v}=q(u+v) H(u, v), \\
H(u, 0)=\frac{1}{2} \int_{0}^{u} q(s) d s, \quad H(0, v)=0 .
\end{gathered}
$$

If the potential $q$ is continuously differentiable, the kernel $K$ itself is the solution of the Goursat problem

$$
\begin{gathered}
\left(\frac{\partial^{2}}{\partial x^{2}}-q(x)\right) K(x, t)=\frac{\partial^{2}}{\partial t^{2}} K(x, t), \\
K(x, x)=\frac{1}{2} \int_{0}^{x} q(s) d s, \quad K(x,-x)=0 .
\end{gathered}
$$

If the potential $q$ is $n$ times continuously differentiable, the kernel $K(x, t)$ is $n+1$ times continuously differentiable with respect to both independent variables (see [25]).

An important property of this transmutation operator consists in the way how it maps solutions of the equation

$$
v^{\prime \prime}+\omega^{2} v=0
$$

into solutions of the equation

$$
u^{\prime \prime}-q(x) u+\omega^{2} u=0
$$

where $\omega$ is a complex number. Denote by $e_{0}(i \omega, x)$ the solution of (8) satisfying the initial conditions

$$
e_{0}(i \omega, 0)=1 \quad \text { and } \quad e_{0}^{\prime}(i \omega, 0)=i \omega
$$

The subindex " 0 " indicates that the initial conditions correspond to the point $x=0$ and the letter " $e$ " reminds us that the initial values coincide with the initial values of the function $e^{i \omega x}$.

The transmutation operator (2) maps $e^{i \omega x}$ into $e_{0}(i \omega, x)$,

$$
e_{0}(i \omega, x)=T\left[e^{i \omega x}\right]
$$

(see [25, Theorem 1.2.1]).

Following [25] we introduce the following notations

$$
K_{c}(x, t ; h)=h+K(x, t)+K(x,-t)+h \int_{t}^{x}\{K(x, \xi)-K(x,-\xi)\} d \xi
$$

where $h$ is a complex number, and

$$
K_{s}(x, t ; \infty)=K(x, t)-K(x,-t) .
$$

Theorem 2 ([25]) Solutions $c(\omega, x ; h)$ and $s(\omega, x ; \infty)$ of equation (8) satisfying the initial conditions

$$
\begin{aligned}
c(\omega, 0 ; h) & =1, & c_{x}^{\prime}(\omega, 0 ; h) & =h \\
s(\omega, 0 ; \infty) & =0, & s_{x}^{\prime}(\omega, 0 ; \infty) & =1
\end{aligned}
$$


can be represented in the form

$$
c(\omega, x ; h)=\cos \omega x+\int_{0}^{x} K_{c}(x, t ; h) \cos \omega t d t
$$

and

$$
s(\omega, x ; \infty)=\frac{\sin \omega x}{\omega}+\int_{0}^{x} K_{s}(x, t ; \infty) \frac{\sin \omega t}{\omega} d t .
$$

Denote by

$$
T_{c} u(x)=u(x)+\int_{0}^{x} K_{c}(x, t ; h) u(t) d t
$$

and

$$
T_{s} u(x)=u(x)+\int_{0}^{x} K_{s}(x, t ; \infty) u(t) d t
$$

the corresponding integral operators. As was pointed out in 3 they are not, in general, transmutations on the whole space $C^{2}[-a, a]$.

\section{$2.2 \quad$ A parametrized family of transmutation operators}

In 3 we introduced the parametrized family of operators $\mathbf{T}_{h}, h \in \mathbb{C}$, given by the integral expression

$$
\mathbf{T}_{h} u(x)=u(x)+\int_{-x}^{x} \mathbf{K}(x, t ; h) u(t) d t
$$

where

$$
\mathbf{K}(x, t ; h)=\frac{h}{2}+K(x, t)+\frac{h}{2} \int_{t}^{x}(K(x, s)-K(x,-s)) d s .
$$

They are related to operators $T_{s}$ and $T_{c}$ (with the parameter $h$ in the kernel of the latter operator) by

$$
\mathbf{T}_{h}=T_{c} P_{e}+T_{s} P_{o}
$$

where $P_{e} f(x)=(f(x)+f(-x)) / 2$ and $P_{o} f(x)=(f(x)-f(-x)) / 2$ are projectors to even and odd functions, respectively. In this subsection we show that the operators $\mathbf{T}_{h}$ are transmutations, summarize their properties and later, in Theorem 15 we show how they act on powers of $x$.

Let us notice that $\mathbf{K}(x, t ; 0)=K(x, t)$ and that the expression

$$
\mathbf{K}(x, t ; h)-\mathbf{K}(x,-t ; h)=K(x, t)-K(x,-t)+\frac{h}{2} \int_{-t}^{t}(K(x, s)-K(x,-s)) d s=K(x, t)-K(x,-t)
$$

does not depend on $h$. Thus, it is possible to compute $\mathbf{K}(x, t ; h)$ for any $h$ by a given $\mathbf{K}\left(x, t ; h_{1}\right)$ for some particular value $h_{1}$. We formulate this result as the following statement.

Theorem 3 ([3]) The integral kernels $\mathbf{K}(x, t ; h)$ and $\mathbf{K}\left(x, t ; h_{1}\right)$ are related by the expression

$$
\mathbf{K}(x, t ; h)=\frac{h-h_{1}}{2}+\mathbf{K}\left(x, t ; h_{1}\right)+\frac{h-h_{1}}{2} \int_{t}^{x}\left(\mathbf{K}\left(x, s ; h_{1}\right)-\mathbf{K}\left(x,-s ; h_{1}\right)\right) d s .
$$

Using (10) let us check how the operators $\mathbf{T}_{h}$ act on solutions of (7).

Proposition 4 The operator $\mathbf{T}_{h}$ maps a solution $v$ of an equation $v^{\prime \prime}+\omega^{2} v=0$, where $\omega$ is a complex number, into the solution $u$ of the equation $u^{\prime \prime}-q(x) u+\omega^{2} u=0$ with the following correspondence of initial values

$$
u(0)=v(0), \quad u^{\prime}(0)=v^{\prime}(0)+h v(0) .
$$


Proof. It follows from (17) and Theorem 2 that the operator $\mathbf{T}_{h}$ indeed maps a solution of $v^{\prime \prime}+\omega^{2} v=$ 0 into a solution of $u^{\prime \prime}-q(x) u+\omega^{2} u=0$. It is clear from the definition (15) that

$$
\mathbf{T}_{h} v(0)=v(0) .
$$

As for the derivative, we have

$$
\left(\mathbf{T}_{h} v\right)^{\prime}(x)=v^{\prime}(x)+\int_{-x}^{x} \mathbf{K}_{x}^{\prime}(x, t ; h) v(t) d t+\mathbf{K}(x, x ; h) v(x)+\mathbf{K}(x,-x ; h) v(-x),
$$

therefore

$$
\left(\mathbf{T}_{h} v\right)^{\prime}(0)=v^{\prime}(0)+K(0,0 ; h) u(0)+K(x,-x ; h) v(0)=v^{\prime}(0)+h v(0) .
$$

Remark 5 As can be seen from the proof, formulas (19) and (20) are valid for any function $v \in$ $C^{1}[-a, a]$.

We know that the kernel of the transmutation operator $T$ is related to the solution of the Goursat problem (3)-(4). We show that a similar result holds for the operators $\mathbf{T}_{h}$.

Theorem 6 In order for the function $K(x, t ; h)$ to be the kernel of a transmutation operator acting as described in Proposition 4, it is necessary and sufficient that $H(u, v ; h):=K(u+v, u-v ; h)$ be a solution of the Goursat problem

$$
\begin{gathered}
\frac{\partial^{2} H(u, v ; h)}{\partial u \partial v}=q(u+v) H(u, v ; h), \\
H(u, 0 ; h)=\frac{h}{2}+\frac{1}{2} \int_{0}^{u} q(s) d s, \quad H(0, v ; h)=\frac{h}{2} .
\end{gathered}
$$

If the potential $q$ is continuously differentiable, the function $K(x, t ; h)$ itself should be the solution of the Goursat problem

$$
\begin{gathered}
\left(\frac{\partial^{2}}{\partial x^{2}}-q(x)\right) K(x, t ; h)=\frac{\partial^{2}}{\partial t^{2}} K(x, t ; h), \\
K(x, x ; h)=\frac{h}{2}+\frac{1}{2} \int_{0}^{x} q(s) d s, \quad K(x,-x ; h)=\frac{h}{2} .
\end{gathered}
$$

Proof. If some transmutation operator $T_{h}$ acts as described in Proposition 4, then on a common domain of definition it coincides with the constructed operator $\mathbf{T}_{h}$, because both operators are continuous and act identically on the dense subset of functions, namely, on linear combinations of solutions of equations (7). Hence it is sufficient to verify that the kernel $\mathbf{K}(x, t ; h)$ satisfies conditions of the theorem.

Suppose $q \in C^{1}[-a, a]$. Then the kernel $K(x, t)$ is twice continuously differentiable and we can check (23) directly. In the following calculation $K_{x}^{\prime}(x, \pm x)$ means $\left.K_{x}^{\prime}(x, t)\right|_{t= \pm x}$ and $K_{t}^{\prime}(x, \pm x)$ means 


$$
\begin{aligned}
& \left.K_{t}^{\prime}(x, t)\right|_{t= \pm x} \\
& \left(\partial_{x}^{2}-\partial_{t}^{2}\right) \mathbf{K}(x, t ; h)=\left(\partial_{x}^{2}-\partial_{t}^{2}\right)\left[\frac{h}{2}+K(x, t)+\frac{h}{2} \int_{t}^{x}(K(x, s)-K(x,-s)) d s\right]= \\
& =q(x) K(x, t)+\frac{h}{2} \partial_{x}\left(\int_{t}^{x}\left(K_{x}^{\prime}(x, s)-K_{x}^{\prime}(x,-s)\right) d s+K(x, x)-K(x,-x)\right)+ \\
& +\frac{h}{2} \partial_{t}(K(x, t)-K(x,-t))=q(x) K(x, t)+\frac{h}{4} q(x)+ \\
& +\frac{h}{2}\left(\int_{t}^{x}\left(K_{x x}^{\prime \prime}(x, s)-K_{x x}^{\prime \prime}(x,-s)\right) d s+K_{x}^{\prime}(x, x)-K_{x}^{\prime}(x,-x)+K_{t}^{\prime}(x, t)+K_{t}^{\prime}(x,-t)\right)= \\
& =q(x) K(x, t)+\frac{h}{4} q(x)+\frac{h}{2}\left(\int_{t}^{x}\left(K_{s s}^{\prime \prime}(x, s)-K_{s s}^{\prime \prime}(x,-s)+q(x)(K(x, s)-K(x,-s))\right) d s+\right. \\
& \left.+K_{x}^{\prime}(x, x)-K_{x}^{\prime}(x,-x)+K_{t}^{\prime}(x, t)+K_{t}^{\prime}(x,-t)\right)=q(x)\left(K(x, t)+\frac{h}{4}+\int_{t}^{x}(K(x, s)-K(x,-s)) d s\right)+ \\
& +\frac{h}{2}\left(K_{t}^{\prime}(x, x)-K_{t}^{\prime}(x, t)+K_{t}^{\prime}(x,-x)-K_{t}^{\prime}(x,-t)+K_{x}^{\prime}(x, x)-K_{x}^{\prime}(x,-x)+K_{t}^{\prime}(x, t)+K_{t}^{\prime}(x,-t)\right)= \\
& =q(x)\left(K(x, t)+\frac{h}{2}+\int_{t}^{x}(K(x, s)-K(x,-s)) d s\right)=q(x) \mathbf{K}(x, t ; h),
\end{aligned}
$$

where we have used (5), (6) and the equalities $K_{x}^{\prime}(x, x)+K_{t}^{\prime}(x, x)=\partial_{x}(K(x, x))=1 / 2 q(x)$, $K_{x}^{\prime}(x,-x)-K_{t}^{\prime}(x,-x)=\partial_{x}(K(x,-x))=0$. Equalities (24) are valid by the definition (16) of $\mathbf{K}(x, t ; h)$. Clearly, the function $\mathbf{H}(u, v ; h)=\mathbf{K}(u+v, u-v ; h)$ is also the solution of the Goursat problem (21)-(22).

Suppose now that $q \in C[-a, a]$. Consider a sequence of functions $\left\{q_{n}\right\}_{n \in \mathbb{N}}$, where $q_{n} \in C^{1}[-a, a]$, $n \in \mathbb{N}$ and $q_{n} \rightarrow q, n \rightarrow \infty$ uniformly on $[-a, a]$. Denote by $H_{n}(u, v)$ the solutions of Goursat problems (3) - (4) with the potentials $q_{n}$, and by $H(u, v)$ the solution with the potential $q$. It is known (see, e.g., [25), that $H_{n}(u, v) \rightarrow H(u, v), n \rightarrow \infty$ uniformly. The same is valid for the functions $K_{n}(x, t)=H_{n}\left(\frac{x+t}{2}, \frac{x-t}{2}\right)$ and $K(x, t)$. Denote by $\mathbf{K}_{n}(x, t ; h)$ the integral kernels constructed from $K_{n}(x, t)$ by means of (16) and let $\mathbf{H}_{n}(u, v ; h)=\mathbf{K}_{n}(u+v, u-v ; h)$. Then $\mathbf{H}_{n}(u, v ; h) \rightarrow \mathbf{H}(u, v ; h)=$ $\mathbf{K}(u+v, u-v ; h), n \rightarrow \infty$ uniformly. Similarly to 25 , 36, the function $\mathbf{H}_{n}(u, v ; h)$ is the solution of the Goursat problem (21)-(22) with the potential $q_{n}$ if and only if $\mathbf{H}_{n}(u, v ; h)$ satisfies the integral equation

$$
\mathbf{H}_{n}(u, v ; h)=\frac{h}{2}+\frac{1}{2} \int_{0}^{u} q_{n}(y) d y+\int_{0}^{u} d \alpha \int_{0}^{v} q_{n}(\alpha+\beta) \mathbf{H}_{n}(\alpha, \beta ; h) d \beta .
$$

From the first part of the proof we know that the functions $\mathbf{H}_{n}(u, v ; h)$ satisfy (25). Passing to the limit when $n \rightarrow \infty$ in (25), we obtain that $\mathbf{H}(u, v ; h)$ satisfies (25) with the potential $q$ and hence satisfies the Goursat problem (21)-(22).

For the second part of the theorem, suppose that a function $H(u, v ; h)$ satisfies the Goursat problem (21)-(22). Consider the Goursat problem (3i)-(41). It is well posed, and from its solution using (16) we can construct the kernel $\mathbf{K}(x, t ; h)$. From the first part of the proof we have that the function $\mathbf{H}(u, v ; h)=\mathbf{K}(u+v, u-v ; h)$ satisfies the Goursat problem (21)-(22), and from the uniqueness of the solution it follows that $H=\mathbf{H}$ and $H$ gives us the transmutation operator acting as described in Proposition 4

Example 7 Consider a function $k(x, t)=\frac{t-1}{2(x+1)}$ (later, in Subsection 3.3 it is explained how it can be obtained). We have

$$
\begin{gathered}
\left(\partial_{x}^{2}-\partial_{t}^{2}\right) k(x, t)=\frac{t-1}{(x+1)^{3}}=\frac{2}{(x+1)^{2}} \cdot \frac{t-1}{2(x+1)} \\
k(x,-x)=\frac{-x-1}{2(x+1)}=-\frac{1}{2} \quad \text { and } \quad k(x, x)=\frac{x-1}{2(x+1)}=-\frac{1}{2}+\frac{1}{2} \int_{0}^{x} \frac{2}{(s+1)^{2}} d s,
\end{gathered}
$$


thus the function $k(x, t)$ satisfies the Goursat problem (23)-(24) with $q(x)=2 /(x+1)^{2}$ and $h=-1$ and by Theorem $\left[\right.$ is the kernel of the transmutation operator $\mathbf{T}_{-1}$.

Consider two solutions of the equation $u^{\prime \prime}=0, u_{1}=1$ and $u_{2}=x$. We have that their images

$$
\begin{aligned}
& v_{1}=\mathbf{T}_{-1} u_{1}=1+\int_{-x}^{x} \frac{t-1}{2(x+1)} d t=\frac{1}{x+1} \\
& v_{2}=\mathbf{T}_{-1} u_{2}=x+\int_{-x}^{x} \frac{(t-1) t}{2(x+1)} d t=\frac{x^{3}+3 x^{2}+3 x}{3(x+1)},
\end{aligned}
$$

are solutions of the equation $v^{\prime \prime}-2 /(x+1)^{2} v=0$ with the initial values in agreement with Proposition 4. For the solution $u_{3}=\cos x$ of the equation $u^{\prime \prime}=-u$ we get that

$$
v_{3}=\mathbf{T}_{-1} u_{3}=\cos x+\int_{-x}^{x} \frac{(t-1) \cos t}{2(x+1)} d t=\cos x-\frac{\sin x}{x+1},
$$

is a solution of the equation $v^{\prime \prime}-\frac{2}{(x+1)^{2}} v=-v$.

For the case $q \in C^{1}[-a, a]$ the Volterra-type integral operator (2) is a transmutation in the sense of Definition 1 on the space $C^{2}[-a, a]$ if and only if the integral kernel $K(x, t)$ satisfies the Goursat problem

$$
\begin{gathered}
\left(\frac{\partial^{2}}{\partial x^{2}}-q(x)\right) K(x, t)=\frac{\partial^{2}}{\partial t^{2}} K(x, t) \\
K(x, x)=C+\frac{1}{2} \int_{0}^{x} q(s) d s, \quad K(x,-x)=C,
\end{gathered}
$$

where $C$ is a constant (see [24, 35]). The proof of this fact consists in differentiating of the integral and integrating by parts, similar to the proof of Theorem 6. Due to Theorem 6 we see that $\mathbf{T}_{h}$ is indeed a transmutation.

Theorem 8 Let $q \in C^{1}[-a, a]$. Then the operator $\mathbf{T}_{h}$ given by (15) satisfies the equality

$$
\left(-\frac{d^{2}}{d x^{2}}+q(x)\right) \mathbf{T}_{h}[u]=\mathbf{T}_{h}\left[-\frac{d^{2}}{d x^{2}}(u)\right]
$$

for any $u \in C^{2}[-a, a]$.

Remark 9 This theorem was proved in [3] under the additional assumption that there exists a nonvanishing solution $f$ of $f^{\prime \prime}-q f=0$ on $[-a, a]$. Later, in Theorem $[26$ we show that such additional assumption allows one to weaken the requirement on the potential $q$ to $q \in C[-a, a]$ keeping valid equality (26).

As can be seen from (15), to define the transmutation operator we have to know its integral kernel in the region $-a<x<a,|t|<|x|$. The corresponding function $H$ should be defined in two domains, upper-right and lower-left triangles $\Pi_{1}: u>0, v>0, u+v<a$ and $\Pi_{3}: u<0, v<0,|u|+|v|<a$ meanwhile the Goursat problem (21) - 22) may be considered in four domains, triangles $\Pi_{1}$ and $\Pi_{3}$ and triangles $\Pi_{2}: u<0, v>0,|u|+|v|<a, \Pi_{4}: u>0, v<0,|u|+|v|<a$. The potential $q$ is continuous in each of these domains, boundary conditions (22) are continuously differentiable, therefore the corresponding solutions of the Goursat problems (see [36]) belong to $C^{1}\left(\bar{\Pi}_{i}\right), i=1 \ldots 4$. Hence the function $\mathbf{H}(u, v ; h)$ is defined in the combined domain $|u|+|v| \leq a$ and belongs to the class $C^{1}$ on it. Consequently, the function $\mathbf{K}(x, t ; h)=\mathbf{H}\left(\frac{x+t}{2}, \frac{x-t}{2} ; h\right)$ is defined on the domain $\bar{\Pi}:-a \leq x \leq a,-a \leq t \leq a$ and is continuously differentiable there. For the rest of this paper we assume that the kernel $\mathbf{K}$ is defined on this larger domain $\bar{\Pi}$. 
Let us find the inverse operator $\mathbf{T}_{h}^{-1}$. Since $\mathbf{T}_{h}$ is the Volterra integral operator its inverse (see [24]) is again a Volterra integral operator

$$
\mathbf{T}_{h}^{-1} u(x)=u(x)+\int_{-x}^{x} L(x, t ; h) u(t) d t,
$$

where $L(x, t ; h)$ satisfies (for $q \in C^{1}[-a, a]$ ) the Goursat problem

$$
\begin{gathered}
\frac{\partial^{2}}{\partial x^{2}} L(x, t ; h)-\left(\frac{\partial^{2}}{\partial t^{2}}-q(t)\right) L(x, t ; h)=0, \\
L(x, x ; h)=C_{1}-\frac{1}{2} \int_{0}^{x} q(s) d s, \quad L(x,-x ; h)=C_{1} .
\end{gathered}
$$

To determine the value of the constant $C_{1}$ we use Proposition 4. Namely, we observe that the integral operator with the kernel $L$ satisfying the Goursat problem (27)-(28) gives us the following correspondence of initial values

$$
v(0)=u(0), \quad v^{\prime}(0)=u^{\prime}(0)+2 C_{1} u(0),
$$

where $v=\mathbf{T}_{h}^{-1} u$. Therefore for the operator to be the inverse of the operator $\mathbf{T}_{h}$ we should take $C_{1}=-h / 2$. Comparing the Goursat problem (27)-(28) with $C_{1}=-h / 2$ with (23)-(24) we conclude that $L(x, t ; h):=-\mathbf{K}(t, x ; h)$ is the unique solution of (27)-(28) and hence it is the kernel of the inverse operator. The assumption $q \in C^{1}[-a, a]$ is not essential and can be easily overcome by considering an approximating sequence of continuously differentiable potentials $q_{n}$. Thus, the following statement is proved.

Theorem 10 The inverse operator $\mathbf{T}_{h}^{-1}$ can be represented as the Volterra integral operator

$$
\mathbf{T}_{h}^{-1} u(x)=u(x)-\int_{-x}^{x} \mathbf{K}(t, x ; h) u(t) d t .
$$

Example 11 For the operator $\mathbf{T}_{-1}$ with the kernel $K(x, t)$ and functions $u_{i}$ and $v_{i}, i=1,2,3$ from Example 7 it is easy to see that indeed

$$
u_{i}(x)=\mathbf{T}_{-1}^{-1}\left[v_{i}\right](x)=v_{i}(x)-\int_{-x}^{x} \frac{x-1}{2(t+1)} v_{i}(t) d t, \quad i=1,2,3 .
$$

\subsection{A complete system of recursive integrals}

Let $f \in C^{2}(a, b) \cap C^{1}[a, b]$ be a complex valued function and $f(x) \neq 0$ for any $x \in[a, b]$. The interval $(a, b)$ is supposed to be finite. Let us consider the following auxiliary functions

$$
\begin{aligned}
\widetilde{X}^{(0)}(x) & \equiv X^{(0)}(x) \equiv 1, \\
\widetilde{X}^{(n)}(x) & =n \int_{x_{0}}^{x} \widetilde{X}^{(n-1)}(s)\left(f^{2}(s)\right)^{(-1)^{n-1}} \mathrm{~d} s, \\
X^{(n)}(x) & =n \int_{x_{0}}^{x} X^{(n-1)}(s)\left(f^{2}(s)\right)^{(-1)^{n}} \mathrm{~d} s,
\end{aligned}
$$

where $x_{0}$ is an arbitrary fixed point in $[a, b]$. We introduce the infinite system of functions $\left\{\varphi_{k}\right\}_{k=0}^{\infty}$ defined as follows

$$
\varphi_{k}(x)= \begin{cases}f(x) X^{(k)}(x), & k \text { odd }, \\ f(x) \widetilde{X}^{(k)}(x), & k \text { even, }\end{cases}
$$

where the definition of $X^{(k)}$ and $\widetilde{X}^{(k)}$ is given by (30)-(32) with $x_{0}$ being an arbitrary point of the interval $[a, b]$. 
Example 12 Let $f \equiv 1, a=0, b=1$. Then it is easy to see that choosing $x_{0}=0$ we have $\varphi_{k}(x)=x^{k}, k \in \mathbb{N}_{0}$ where by $\mathbb{N}_{0}$ we denote the set of non-negative integers.

In [19] it was shown that the system $\left\{\varphi_{k}\right\}_{k=0}^{\infty}$ is complete in $L_{2}(a, b)$ and in [20] its completeness in the space of piecewise differentiable functions with respect to the maximum norm was obtained and series expansions in terms of the functions $\varphi_{k}$ were studied.

The system (33) is closely related to the notion of the $L$-basis introduced and studied in [8]. Here the letter $L$ corresponds to a linear ordinary differential operator. This becomes more transparent from the following result obtained in [17] (for additional details and simpler proof see [18] and [21]) establishing the relation of the system of functions $\left\{\varphi_{k}\right\}_{k=0}^{\infty}$ to Sturm-Liouville equations.

Theorem 13 ([17]) Let $q$ be a continuous complex valued function of an independent real variable $x \in[a, b], \lambda$ be an arbitrary complex number. Suppose there exists a solution $f$ of the equation

$$
f^{\prime \prime}-q f=0
$$

on $(a, b)$ such that $f \in C^{2}(a, b)$ together with $1 / f$ are bounded on $[a, b]$. Then the general solution of the equation

$$
u^{\prime \prime}-q u=\lambda u
$$

on $(a, b)$ has the form

$$
u=c_{1} u_{1}+c_{2} u_{2}
$$

where $c_{1}$ and $c_{2}$ are arbitrary complex constants,

$$
u_{1}=\sum_{k=0}^{\infty} \frac{\lambda^{k}}{(2 k) !} \varphi_{2 k} \quad \text { and } \quad u_{2}=\sum_{k=0}^{\infty} \frac{\lambda^{k}}{(2 k+1) !} \varphi_{2 k+1}
$$

and both series converge uniformly on $[a, b]$.

Remark 14 It is easy to see that by definition the solutions $u_{1}$ and $u_{2}$ satisfy the following initial conditions

$$
\begin{array}{cc}
u_{1}\left(x_{0}\right)=f\left(x_{0}\right), & u_{1}^{\prime}\left(x_{0}\right)=f^{\prime}\left(x_{0}\right), \\
u_{2}\left(x_{0}\right)=0, & u_{2}^{\prime}\left(x_{0}\right)=1 / f\left(x_{0}\right) .
\end{array}
$$

\subsection{Transmutations and systems of recursive integrals}

In this subsection we show the connection between the transmutation operators $\mathbf{T}_{h}$ and the functions $\varphi_{k}$. All the results of this subsection were proved in $[3$. Here we recall them with explanations.

We suppose that $f$ is a solution of (34) fulfilling the condition of Theorem 13 on a finite interval $(-a, a)$. We normalize $f$ in such a way that $f(0)=1$, and let $f^{\prime}(0)=h$ where $h$ is some complex constant. Let us obtain the expansion of the solution $c(\omega, x ; h)$ from Subsection 2.1 in terms of the functions $\varphi_{k}$. According to Remark 14 the solutions (36) of equation (35) have the following initial values

$$
u_{1}(0)=1, \quad u_{1}^{\prime}(0)=h, \quad u_{2}(0)=0, \quad u_{2}^{\prime}(0)=1 .
$$

Hence due to (11) we obtain $c(\omega, x ; h)=u_{1}(x)$. From (13) and (36) we have the equality

$$
\sum_{k=0}^{\infty} \frac{(i \omega)^{2 k}}{(2 k) !} \varphi_{2 k}(x)=\sum_{j=0}^{\infty} \frac{(i \omega)^{2 j} x^{2 j}}{(2 j) !}+\int_{0}^{x}\left(K_{c}(x, t ; h) \sum_{j=0}^{\infty} \frac{(i \omega)^{2 j} t^{2 j}}{(2 j) !}\right) d t .
$$

As the series under the sign of integral converges uniformly and the kernel $K_{c}(x, t ; h)$ is at least continuously differentiable (for a continuous $q$ [25]) we obtain the following relation

$$
\sum_{k=0}^{\infty} \frac{(i \omega)^{2 k}}{(2 k) !} \varphi_{2 k}(x)=\sum_{j=0}^{\infty} \frac{(i \omega)^{2 j}}{(2 j) !}\left(x^{2 j}+\int_{0}^{x} K_{c}(x, t ; h) t^{2 j} d t\right) .
$$


This equality holds for any $\omega$ hence we obtain the termwise relations

$$
\varphi_{2 k}=T_{c}\left[x^{2 k}\right], \quad k \in \mathbb{N}_{0} .
$$

Similarly, due to (12) we observe that $s(\omega, x ; \infty)=u_{2}(x)$ and from (14) we find that

$$
\varphi_{2 k+1}=T_{s}\left[x^{2 k+1}\right], \quad k \in \mathbb{N}_{0} .
$$

Hence from the last two equalities and (17) we conclude that the following statement is true.

Theorem 15 ([3]) Let $q$ be a continuous complex valued function of an independent real variable $x \in[-a, a]$, and $f$ be a particular solution of (34) such that $f \in C^{2}(-a, a)$ together with $1 / f$ are bounded on $[-a, a]$ and normalized as $f(0)=1, f^{\prime}(0)=h$, where $h$ is a complex number. Then the operator (15) with the kernel defined by (16) transforms $x^{k}$ into $\varphi_{k}(x)$ for any $k \in \mathbb{N}_{0}$.

Thus, we clarified what is the result of application of the transmutation $\mathbf{T}_{h}$ to the powers of the independent variable. This is very useful due to the fact that as a rule the construction of the kernel $\mathbf{K}(x, t ; h)$ in a more or less explicit form up to now is impossible. Our result offers an algorithm for transmuting functions which can be represented or at least approximated by finite or infinite polynomials in the situation when $\mathbf{K}(x, t ; h)$ is unknown.

Remark 16 Let $f$ be the solution of (34) satisfying the initial conditions

$$
f(0)=1 \quad \text { and } \quad f^{\prime}(0)=0 .
$$

If it does not vanish on $[-a, a]$ then from Theorem 15 we obtain that the original transmutation operator $T$ transmutes powers of the independent variable into $\varphi_{k}: \varphi_{k}=T\left[x^{k}\right]$ for any $k \in \mathbb{N}_{0}$. In general, of course $f$ may have zeros on $[-a, a]$ and hence one can guarantee that the operator $T$ transmutes the powers of $x$ into $\varphi_{k}$ whose construction is based on the solution $f$ satisfying (41) only in some neighborhood of the origin. If we construct $\varphi_{k}$ by a solution $f$ such that $f(0)=1$ and $f^{\prime}(0)=h$, then the original transmutation operator $T$ does not map powers of the independent variable exactly to the functions $\varphi_{k}$. As it was shown in [3] the following equalities are valid

$$
\begin{aligned}
\varphi_{k} & =T\left[x^{k}\right] \quad \text { when } k \text { is odd, } \\
\varphi_{k}-\frac{h}{k+1} \varphi_{k+1} & =T\left[x^{k}\right] \quad \text { when } k \in \mathbb{N}_{0} \text { is even. }
\end{aligned}
$$

This observation explains the necessity to consider the parametrized family of transmutation operators whose mapping properties are well adjusted to corresponding families of the functions $\varphi_{k}$.

Example 17 For the operator $\mathbf{T}_{-1}$ from Example 7 , consider the function $f=\mathbf{T}_{-1}[1]=\frac{1}{x+1}$ as a solution of (34) such that $f(0)=1$ and $f^{\prime}(0)=h=-1$, nonvanishing on any $[-a, a] \subset(-1,1)$. The first 3 functions $\varphi_{k}$ are given by

$$
\varphi_{0}=f=\frac{1}{x+1}, \quad \varphi_{1}=\frac{x^{3}+3 x^{2}+3 x}{3(x+1)}, \quad \varphi_{2}=\frac{2 x^{3}+3 x^{2}}{3(x+1)} .
$$

We have seen already that $\varphi_{0}=\mathbf{T}_{-1}[1]$ and $\varphi_{1}=\mathbf{T}_{-1}[x]$. We have also $\mathbf{T}_{-1}\left[x^{2}\right]=x^{2}-\frac{x^{3}}{3(x+1)}=\varphi_{2}$.

\section{Transmutation operators for Darboux transformed equa- tions}

\subsection{The Darboux transformation}

Consider a Sturm-Liouville operator $A_{1}:=\frac{d^{2}}{d x^{2}}-q_{1}(x)$, where $q_{1}$ is a continuous complex-valued function on some segment $[-a, a]$. Suppose a solution $f$ of the equation $A_{1} f=0$ is given such that 
$f(x) \neq 0, x \in[-a, a]$, it is normalized as $f(0)=1$ and $h:=f^{\prime}(0)$ is some complex number. Then the operator $A_{2}:=\frac{d^{2}}{d x^{2}}-q_{2}(x)$, where $q_{2}(x)=2\left(\frac{f^{\prime}(x)}{f(x)}\right)^{2}-q_{1}(x)$, is known as the Darboux transformation of the operator $A_{1}$.

Initially the Darboux transformation served for establishing a relation between the general solution $u$ of the equation $A_{1} u=\lambda u$ and the general solution $v$ of the equation $A_{2} v=\lambda v$ by a known particular solution $f$ of the equation $A_{1} u=0$. This relation is given by the formula $v(x)=u^{\prime}(x)-u(x) \frac{f^{\prime}(x)}{f(x)}$. Later on it was found that the Darboux transformation was closely related to the factorization of the Schrödinger equation, and nowadays it is used in dozens of works, see e.g. [7, 10, 26, 30, in connection with solitons and integrable systems, e.g. [1, 11, 28, 29] and the review [31] of applications to quantum mechanics.

We remind some well known facts about the Darboux transformation. First, $1 / f$ is the solution of $A_{2} u=0$. Second, it is closely related to the factorization of Sturm-Liouville and one-dimensional Schrödinger operators. Namely, we have

$$
\begin{aligned}
& A_{1}=\frac{d^{2}}{d x^{2}}-q_{1}(x)=\left(\partial_{x}+\frac{f^{\prime}}{f}\right)\left(\partial_{x}-\frac{f^{\prime}}{f}\right)=\frac{1}{f} \partial_{x} f^{2} \partial_{x} \frac{1}{f} \cdot \\
& A_{2}=\frac{d^{2}}{d x^{2}}-q_{2}(x)=\left(\partial_{x}-\frac{f^{\prime}}{f}\right)\left(\partial_{x}+\frac{f^{\prime}}{f}\right)=f \partial_{x} \frac{1}{f^{2}} \partial_{x} f \cdot .
\end{aligned}
$$

Suppose that $u$ is a solution of the equation $A_{1} u=\omega u$ for some $\omega \in \mathbb{C}$. Then the function $v=$ $\left(\partial_{x}-\frac{f^{\prime}}{f}\right) u=\left(f \partial_{x} \frac{1}{f}\right) u$ is a solution of the equation $A_{2} v=\omega v$, and vice versa, given a solution $v$ of $A_{2} v=\omega v$, the function $u=\left(\partial_{x}+\frac{f^{\prime}}{f}\right) v=\left(\frac{1}{f} \partial_{x} f\right) v$ is a solution of $A_{1} u=\omega u$.

\subsection{Construction of transmutation operators for Darboux transformed equations}

Consider the operators $A_{1}, A_{2}$ and a particular solution $f$ of the equation $A_{1} f=0$ as in the previous subsection. Suppose that the operator $\mathbf{T}_{1 ; h}$ which transmutes the operator $A_{1}$ into the operator $B=d^{2} / d x^{2}$ is known in the sense that its kernel $\mathbf{K}_{1}(x, t ; h)$ is given. As before $h=f^{\prime}(0)$ and $\mathbf{T}_{1 ; h}$ transforms solutions according to Proposition 4. As the parameter $h$ is fixed by the value $f^{\prime}(0)$ we will simply write $\mathbf{T}_{1}$ instead of $\mathbf{T}_{1 ; h}$. We know that the function $1 / f$ is the non-vanishing solution of the equation $A_{2} u=0$ satisfying $1 / f(0)=1$ and $(1 / f)^{\prime}(0)=-h$. Hence it is natural to look for the operator $\mathbf{T}_{2 ;-h}$ transmuting the operator $A_{2}$ into the operator $B$. We will simply write $\mathbf{T}_{2}$ further in this paper.

Let us explain the idea for obtaining the operator $\mathbf{T}_{2}$. We want to find an operator transforming solutions of the equation $B u+\omega^{2} u=0$ into solutions of the equation $A_{2} u+\omega^{2} u=0$, see the first diagram below. Starting with a solution $\sigma$ of the equation $\left(\partial_{x}^{2}+\omega^{2}\right) \sigma=0$, by application of $\mathbf{T}_{1}$ we get a solution of $\left(A_{1}+\omega^{2}\right) u=0$, and the expression $\left(f \partial_{x} \frac{1}{f}\right) \mathbf{T}_{1} \sigma$ is a solution of $\left(A_{2}+\omega^{2}\right) v=0$. But the operator $\left(f \partial_{x} \frac{1}{f}\right) \mathbf{T}_{1}$ is unbounded and hence cannot coincide with the operator $\mathbf{T}_{2}$. In order to find the required bounded operator we may consider the second copy of the equation $\left(\partial_{x}^{2}+\omega^{2}\right) u=0$, which is a result of the Darboux transformation applied to $\left(\partial_{x}^{2}+\omega^{2}\right) \sigma=0$ with respect to the particular solution $g \equiv 1$ and construct the operator $\mathbf{T}_{2}$ by making the second diagram commutative. In order to obtain a bounded operator $\mathbf{T}_{2}$, instead of using $f \partial_{x} \frac{1}{f}$ for the last step, we will use the inverse of $\frac{1}{f} \partial_{x} f$, i.e. $\frac{1}{f}\left(\int_{0}^{x} f(s) \cdot d s+C\right)$.
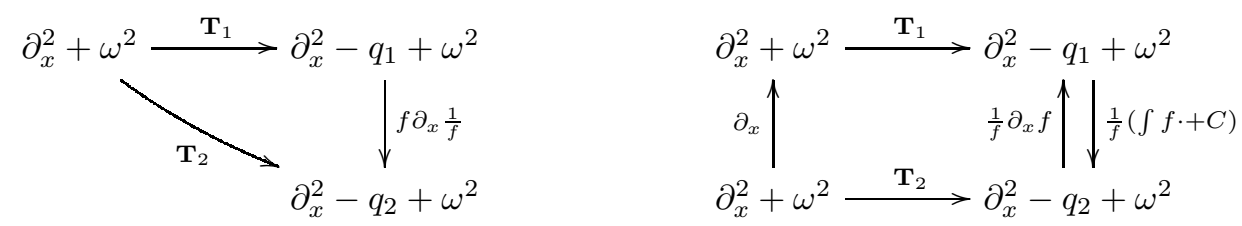
That explains how to obtain the following theorem.

Theorem 18 The operator $T_{2}$, acting on solutions $u$ of equations $\left(\partial_{x}^{2}+\omega^{2}\right) u=0, \omega \in \mathbb{C}$ by the rule

$$
T_{2}[u](x)=\frac{1}{f(x)}\left(\int_{0}^{x} f(\eta) \mathbf{T}_{1}\left[u^{\prime}\right](\eta) d \eta+u(0)\right)
$$

coincides with the transmutation operator $\mathbf{T}_{2 ;-h}$.

Proof. According to Proposition 4 the operator $\mathbf{T}_{2 ;-h}$ transforms a solution $u$ of the equation $\left(\partial_{x}^{2}+\omega^{2}\right) u=0$ into a solution of the equation $\left(A_{2}+\omega^{2}\right) v=\left(\partial_{x}^{2}-q_{2}(x)+\omega^{2}\right) v=0$ with the correspondence of initial conditions

$$
\mathbf{T}_{2 ;-h}[u](0)=u(0), \quad\left(\mathbf{T}_{2 ;-h}[u]\right)^{\prime}(0)=u^{\prime}(0)-h u(0) .
$$

Hence we have to verify the same properties for the operator $T_{2}$. Consider a solution $u$ of the equation $\left(\partial_{x}^{2}+\omega^{2}\right) u=0$ for some particular $\omega \in \mathbb{C}$. The function $u^{\prime}$ is again a solution of this equation, and by Proposition 4, $\mathbf{T}_{1} u$ is a solution of the equation $\left(\partial_{x}^{2}-q_{1}+\omega^{2}\right) v=0$, so with the aid of the factorization (42) we obtain

$$
\frac{1}{f} \partial_{x} f^{2} \partial_{x} \frac{1}{f} \mathbf{T}_{1}\left[u^{\prime}\right]=-\omega^{2} \mathbf{T}_{1}\left[u^{\prime}\right]
$$

Let us apply the operator $A_{2}$ to the function $T_{2} u$ and use the factorization (43).

$$
A_{2} T_{2}[u](x)=\left(f \partial_{x} \frac{1}{f^{2}} \partial_{x} f\right)\left(\frac{1}{f(x)}\left(\int_{0}^{x} f(s) \mathbf{T}_{1}\left[u^{\prime}\right](s) d s+u(0)\right)\right)=\left(f \partial_{x} \frac{1}{f}\right)\left(\mathbf{T}_{1}\left[u^{\prime}\right]\right) .
$$

Applying $\frac{1}{f} \partial_{x} f$ to both sides of (47) due to (46) we obtain

$$
\left(\frac{1}{f} \partial_{x} f\right)\left(A_{2} T_{2}[u]\right)=\left(\frac{1}{f} \partial_{x} f^{2} \partial_{x} \frac{1}{f}\right)\left(\mathbf{T}_{1}\left[u^{\prime}\right]\right)=-\omega^{2} \mathbf{T}_{1}\left[u^{\prime}\right]=-\omega^{2}\left(\frac{1}{f} \partial_{x} f\right)\left(T_{2}[u]\right) .
$$

Hence the function $A_{2} T_{2}[u]$ may differ from the function $-\omega^{2} T_{2}[u]$ only by $c / f$, where $c$ is a constant. To find the value of the constant we compute the values of both expressions for $x=0$. We have

$$
T_{2}[u](0)=u(0)
$$

and

$$
A_{2} T_{2}[u](x)=\left(f \partial_{x} \frac{1}{f}\right)\left(\mathbf{T}_{1}\left[u^{\prime}\right](x)\right)=-\frac{f^{\prime}(x)}{f(x)} \mathbf{T}_{1}\left[u^{\prime}\right](x)+\left(\mathbf{T}_{1}\left[u^{\prime}\right](x)\right)^{\prime} .
$$

As $u^{\prime}$ is a solution of $\left(\partial_{x}^{2}+\omega^{2}\right) v=0$, by Proposition 4 we have $\mathbf{T}_{1}\left[u^{\prime}\right](0)=u^{\prime}(0),\left(\mathbf{T}_{1}\left[u^{\prime}\right]\right)^{\prime}(0)=$ $u^{\prime \prime}(0)+h u^{\prime}(0)=-\omega^{2} u(0)+h u^{\prime}(0)$ (the last equality holds since the function $u$ is also a solution of $\left.\left(\partial_{x}^{2}+\omega^{2}\right) v=0\right)$. Therefore

$$
A_{2} T_{2}[u](0)=-h u^{\prime}(0)+\omega u(0)+h u^{\prime}(0)=-\omega^{2} u(0)
$$

Hence $c=0$ and the operator $T_{2}$ maps solutions of the equation $\left(\partial_{x}^{2}+\omega^{2}\right) u=0$ into solutions of the equation $\left(A_{2}+\omega^{2}\right) v=0$ for any $\omega \in \mathbb{C}$. To finish the proof, we have to check conditions (45) for the operator $T_{2}$. We have

$$
T_{2}[u](0)=\frac{u(0)}{f(0)}=u(0)
$$

and

$$
\left(T_{2}[u]\right)^{\prime}(x)=-\frac{f^{\prime}(x)}{f(x)} T_{2}[u](x)+\frac{1}{f(x)} \cdot f(x) \mathbf{T}_{1}\left[u^{\prime}\right](x)=-\frac{f^{\prime}(x)}{f(x)} T_{2}[u](x)+\mathbf{T}_{1}\left[u^{\prime}\right](x)
$$


hence for $x=0$ we obtain

$$
\left(T_{2}[u]\right)^{\prime}(0)=-h T_{2}[u](0)+\mathbf{T}_{1}\left[u^{\prime}\right](0)=-h u(0)+u^{\prime}(0),
$$

which finishes the proof.

Now we show that the operator $T_{2}$ can be written as a Volterra integral operator and, as a consequence, extended by continuity to a wider class of functions.

Theorem 19 The operator $T_{2}$ admits a representation as the Volterra integral operator

$$
T_{2}[u](x)=u(x)+\int_{-x}^{x} \mathbf{K}_{2}(x, t ;-h) u(t) d t,
$$

with the kernel

$$
\mathbf{K}_{2}(x, t ;-h)=-\frac{1}{f(x)}\left(\int_{-t}^{x} \partial_{t} \mathbf{K}_{1}(s, t ; h) f(s) d s+\frac{h}{2} f(-t)\right) .
$$

Proof. Consider the expression

$$
\int_{0}^{x} f(t) \mathbf{T}_{1}\left[u^{\prime}\right](t) d t+u(0)=\int_{0}^{x} f(t) u^{\prime}(t) d t+\int_{0}^{x} f(t) \int_{-t}^{t} \mathbf{K}_{1}(t, s ; h) u^{\prime}(s) d s d t+u(0) .
$$

Suppose that $x>0$ (the opposite case is similar). We integrate by parts the first integral and change the order of integration in the second integral,

$$
\begin{gathered}
\int_{0}^{x} f(t) \mathbf{T}_{1}\left[u^{\prime}\right](t) d t+u(0)= \\
f(x) u(x)-f(0) u(0)-\int_{0}^{x} f^{\prime}(t) u(t) d t+\int_{-x}^{x} u^{\prime}(s) \int_{|s|}^{x} \mathbf{K}_{1}(t, s ; h) f(t) d t d s+u(0)= \\
f(x) u(x)-\int_{0}^{x} f^{\prime}(t) u(t) d t+\left.u(s) \int_{|s|}^{x} \mathbf{K}_{1}(t, s ; h) f(t) d t\right|_{-x} ^{x}-\int_{-x}^{x} u(s) \frac{d}{d s}\left(\int_{|s|}^{x} \mathbf{K}_{1}(t, s ; h) f(t) d t\right) d s= \\
f(x) u(x)-\int_{0}^{x} f^{\prime}(t) u(t) d t-\int_{-x}^{x} u(s) \frac{d}{d s}\left(\int_{|s|}^{x} \mathbf{K}_{1}(t, s ; h) f(t) d t\right) d s . \quad(50)
\end{gathered}
$$

To continue the proof we will use the extension of the kernel $\mathbf{K}_{1}$ onto the square $|x| \leq a,|t| \leq a$ as a continuously differentiable function. Consider the integral

$$
\int_{-s}^{|s|} \mathbf{K}_{1}(t, s ; h) f(t) d t
$$

For $s \leq 0$ it equals zero. For $s>0$ note that by Theorem[15, $\mathbf{T}_{1}[1](x)=f(x)$, so we obtain from (29)

$$
1=\mathbf{T}_{1}^{-1}[f](x)=f(x)-\int_{-x}^{x} \mathbf{K}_{1}(t, x ; h) f(t) d t .
$$

Hence

$$
\int_{-s}^{|s|} \mathbf{K}_{1}(t, s ; h) f(t) d t= \begin{cases}0, & \text { if } s \leq 0 \\ f(s)-1, & \text { if } s>0\end{cases}
$$


Combining (50) and (51), we get

$$
\begin{gathered}
\int_{0}^{x} f(t) \mathbf{T}_{1}\left[u^{\prime}\right](t) d t+u(0)=f(x) u(x)-\int_{0}^{x} f^{\prime}(t) u(t) d t- \\
-\int_{-x}^{x} u(s) \frac{d}{d s}\left(\int_{-s}^{x} \mathbf{K}_{1}(t, s ; h) f(t) d t-\int_{-s}^{|s|} \mathbf{K}_{1}(t, s ; h) f(t) d t\right) d s= \\
=f(x) u(x)-\int_{0}^{x} f^{\prime}(t) u(t) d t-\int_{-x}^{x} u(s) \frac{d}{d s}\left(\int_{-s}^{x} \mathbf{K}_{1}(t, s ; h) f(t) d t\right) d s+\int_{0}^{x} u(s) f^{\prime}(s) d s= \\
=f(x) u(x)-\int_{-x}^{x} u(s)\left(\int_{-s}^{x} \frac{d}{d s} \mathbf{K}_{1}(t, s ; h) f(t) d t+\mathbf{K}_{1}(-s, s ; h) f(-s)\right) d s= \\
\quad=f(x) u(x)-\int_{-x}^{x} u(s)\left(\int_{-s}^{x} \frac{d}{d s} \mathbf{K}_{1}(t, s ; h) f(t) d t+\frac{h}{2} f(-s)\right) d s .
\end{gathered}
$$

Hence

$$
T_{2}[u](x)=\frac{1}{f(x)}\left(\int_{0}^{x} f(\eta) \mathbf{T}_{1}\left[u^{\prime}\right](\eta) d \eta+u(0)\right)=u(x)+\int_{-x}^{x} \mathbf{K}_{2}(x, t ;-h) u(t) d t,
$$

where the kernel $\mathbf{K}_{2}$ is given by (49).

Remark 20 Note that in the proof of Theorem 19 we made use only of the fact that $u \in C^{1}[-a, a]$ and never required that $u$ be a solution of the equation $\partial_{x}^{2} u+\omega^{2} u=0$. Therefore both representations for the operator $T_{2}$ obtained in Theorems 18 and 19 coincide on any function $u \in C^{1}[-a, a]$.

Remark 21 If the integral kernel $\mathbf{K}_{1}$ is known only in the domain $|x| \leq a,|t| \leq|x|$, from (50) it is also possible to obtain the expression of the integral kernel for the Volterra integral operator representing $T_{2}$. Since

$$
\begin{aligned}
\frac{d}{d t}\left(\int_{|t|}^{x} \mathbf{K}_{1}(s, t ; h) f(s) d s\right)= & \int_{|t|}^{x} \frac{d}{d t} \mathbf{K}_{1}(s, t ; h) f(s) d s-\mathbf{K}_{1}(|t|, t ; h) f(|t|) \cdot(|t|)^{\prime}= \\
& = \begin{cases}\int_{|t|}^{x} \frac{d}{d t} \mathbf{K}_{1}(s, t ; h) f(s) d s-\frac{h}{2} f(t)-\frac{f(t)}{2} \int_{0}^{t} q_{1}(s) d s & \text { for } t \geq 0, \\
\int_{|t|}^{x} \frac{d}{d t} \mathbf{K}_{1}(s, t ; h) f(s) d s+\frac{h}{2} f(-t) & \text { for } t<0,\end{cases}
\end{aligned}
$$

the integral kernel for $x>0$ is given by the expression

$$
\mathbf{K}_{2}(x, t ;-h)= \begin{cases}-\frac{1}{f(x)}\left(f^{\prime}(t)+\int_{|t|}^{x} \frac{d}{d t}\left(\mathbf{K}_{1}(s, t ; h)\right) f(s) d s-\frac{h}{2} f(t)-\frac{f(t)}{2} \int_{0}^{t} q_{1}(s) d s\right) & \text { if } t \geq 0, \\ -\frac{1}{f(x)}\left(\int_{|t|}^{x} \frac{d}{d t}\left(\mathbf{K}_{1}(s, t ; h)\right) f(s) d s+\frac{h}{2} f(-t)\right) & \text { if } t<0 .\end{cases}
$$

Corollary 22 The operator $T_{2}$ given by (48) with the kernel (49) coincides with $\mathbf{T}_{2}$ on $C[-a, a]$.

Proof. By Theorems 18 and 19 the Volterra operators $T_{2}$ and $\mathbf{T}_{2}$ coincide on the set of finite linear combinations of solutions of the equations $\left(\partial_{x}^{2}+\omega^{2}\right) u=0, \omega \in \mathbb{C}$. Since this set is dense in $C[-a, a]$, by continuity of $T_{2}$ and $\mathbf{T}_{2}$ we obtain that they coincide on the whole $C[-a, a]$.

The next corollary follows immediately from Remark 20.

Corollary 23 The operator $T_{2}$ given by (44) coincides with $\mathbf{T}_{2}$ on $C^{1}[-a, a]$.

Operator $A_{1}$ is the Darboux transformation of the operator $A_{2}$ with respect to the solution $1 / f$, hence we obtain another relation between the operators $\mathbf{T}_{1}$ and $\mathbf{T}_{2}$. 
Corollary 24 For any function $u \in C^{1}[-a, a]$ the equality

$$
\mathbf{T}_{1}[u](x)=f(x)\left(\int_{0}^{x} \frac{1}{f(\eta)} \mathbf{T}_{2}\left[u^{\prime}\right](\eta) d \eta+u(0)\right)
$$

is valid.

From the second commutative diagram at the beginning of this subsection we may deduce some commutation relations between the operators $\mathbf{T}_{1}, \mathbf{T}_{2}$ and $d / d x$. The proof immediately follows from (44) and (52).

Corollary 25 The following operator equalities hold on $C^{1}[-a, a]$ :

$$
\begin{aligned}
\partial_{x} f \mathbf{T}_{2} & =f \mathbf{T}_{1} \partial_{x} \\
\partial_{x} \frac{1}{f} \mathbf{T}_{1} & =\frac{1}{f} \mathbf{T}_{2} \partial_{x} .
\end{aligned}
$$

Commutation relations (53) and (54) allow us to prove a more general version of [3, Theorem 11] which is also Theorem 8 with different conditions on the potential $q$.

Theorem 26 Under the conditions of Theorem [15 the operator (15) with the kernel defined by (16) satisfies

$$
\left(-\frac{d^{2}}{d x^{2}}+q(x)\right) \mathbf{T}_{h}[u]=\mathbf{T}_{h}\left[-\frac{d^{2} u}{d x^{2}}\right]
$$

for any $u \in C^{2}[-a, a]$.

Proof. Let $\mathbf{T}_{2}$ be the transmutation operator of the Darboux transformation of the operator $A=$ $-\frac{d^{2}}{d x^{2}}+q(x)$ with respect to the solution $f$. Let $u \in C^{2}[-a, a]$, then $u^{\prime} \in C^{1}[-a, a]$ and we obtain by (53), (54) and (42)

$$
\mathbf{T}_{h}\left[\frac{d^{2} u}{d x^{2}}\right]=\frac{1}{f} \frac{d}{d x}\left[f \mathbf{T}_{2} u^{\prime}\right]=\frac{1}{f} \frac{d}{d x} f^{2} \frac{d}{d x} \frac{1}{f} \mathbf{T}_{h} u=\left(\frac{d^{2}}{d x^{2}}-q(x)\right) \mathbf{T}_{h} u .
$$

In 20] the following notion of generalized derivatives was introduced. Consider a function $g$ assuming that both $f$ and $g$ possess the derivatives of all orders up to the order $n$ on the segment $[-a, a]$. Then in $[-a, a]$ the following generalized derivatives are defined

$$
\begin{aligned}
& \gamma_{0}(g)(x)=g(x), \\
& \gamma_{k}(g)(x)=\left(f^{2}(x)\right)^{(-1)^{k-1}}\left(\gamma_{k-1}(g)\right)^{\prime}(x)
\end{aligned}
$$

for $k=1,2, \ldots, n$.

Let a function $u$ be defined by the equality

$$
g=\frac{1}{f} \mathbf{T}_{1} u
$$

and assume that $u \in C^{n}[-a, a]$. Note that below we do not necessarily require that the functions $f$ and $g$ be from $C^{n}[-a, a]$. With the use of (53) and (54) we have

$$
\begin{aligned}
& \gamma_{1}(g)=f^{2} \cdot\left(\frac{1}{f} \mathbf{T}_{1} u\right)^{\prime}=f^{2} \cdot \frac{1}{f} \mathbf{T}_{2} u^{\prime}=f \mathbf{T}_{2} u^{\prime}, \\
& \gamma_{2}(g)=\frac{1}{f^{2}} \cdot\left(f \mathbf{T}_{2} u^{\prime}\right)^{\prime}=\frac{1}{f^{2}} \cdot f \mathbf{T}_{1} u^{\prime \prime}=\frac{1}{f} \mathbf{T}_{1} u^{\prime \prime} .
\end{aligned}
$$

By induction we obtain the following corollary. 
Corollary 27 Let $u \in C^{n}[-a, a]$ and $g=\frac{1}{f} \mathbf{T}_{1} u$. Then

$$
\gamma_{k}(g)=f \mathbf{T}_{2} u^{(k)} \quad \text { if } k \text { is odd, } k \leq n,
$$

and

$$
\gamma_{k}(g)=\frac{1}{f} \mathbf{T}_{1} u^{(k)} \quad \text { if } k \text { is even, } k \leq n .
$$

To finish this subsection, let us consider how the operator $\mathbf{T}_{2 ;-}$ acts on powers of the independent variable. By Theorem 15 we have to construct the system of functions $\left\{\varphi_{k}\right\}$ by formulas (30)-(32) and (33) starting with the function $1 / f$. As it can be seen from (30)-(32), for any nonnegative integer $k$ we have

$$
\tilde{X}_{1 / f}^{(k)}=X_{f}^{(k)} \quad \text { and } \quad X_{1 / f}^{(k)}=\widetilde{X}_{f}^{(k)},
$$

where the subindex $f$ or $1 / f$ corresponds to the starting function used in (30)-(32). Therefore constructing the functions $X^{(k)}, \widetilde{X}^{(k)}$ according to (30)-(32) and defining

$$
\psi_{k}(x)= \begin{cases}\frac{1}{f(x)} X^{(k)}, & k \text { even, } \\ \frac{1}{f(x)} \widetilde{X}^{(k)}, & k \text { odd },\end{cases}
$$

(here the "second half" of the formal powers (30)-(32) is used, cf. (33)) we obtain the following statement.

Proposition 28 Let a potential $q_{1}$ and a function $f$ be as in Theorem 15. Let the operator $\mathbf{T}_{2 ;-h}$ be the transmutation operator for the Darboux transformed operator $A_{2}$. Then $\mathbf{T}_{2 ;-h}$ transforms $x^{k}$ into $\psi_{k}(x)$ for any $k \in \mathbb{N}_{0}$.

\subsection{Examples}

We start with the operator $A_{0}=d^{2} / d x^{2}$. We have to pick up such a solution $f$ of the equation $A_{0} f=0$ that $f^{\prime} / f \neq 0$. This is in order to obtain an operator $A_{1} \neq A_{0}$ as a result of the Darboux transformation of $A_{0}$. As such solution consider, e.g., $f_{0}(x)=x+1$. Both $f_{0}$ and $1 / f_{0}$ are bounded on any segment $[-a, a] \subset(-1 ; 1)$ and the Darboux transformed operator has the form $A_{1}=\frac{d^{2}}{d x^{2}}-\frac{2}{(x+1)^{2}}$.

The transmutation operator $T$ for $A_{0}$ is obviously an identity operator and $K_{0}(x, t ; 0)=0$. Since $f_{0}^{\prime}(0)=1$, we look for the parametrized operator $\mathbf{T}_{0 ; 1}$. Its kernel is given by (18): $\mathbf{K}_{0}(x, t ; 1)=1 / 2$. From Theorem 19 we obtain the transmutation kernel for the operator $A_{1}$

$$
\mathbf{K}_{1}(x, t ;-1)=-\frac{1}{x+1} \cdot \frac{1-t}{2}=\frac{t-1}{2(x+1)},
$$

the kernel from Example 7

To obtain a less trivial example consider again the operator $A_{1}=\frac{d^{2}}{d x^{2}}-\frac{2}{(x+1)^{2}}$ and the function $f_{1}(x)=(x+1)^{2}$ as a solution of $A_{1} f=0$. Since $h=f_{1}^{\prime}(0)=2$, we compute $\mathbf{K}_{1}(x, t ; 2)$ from (56) using (18)

$$
\mathbf{K}_{1}(x, t ; 2)=\frac{3 x^{2}+6 x+4-3 t^{2}+2 t}{4(x+1)} .
$$

The Darboux transformation of the operator $A_{1}$ with respect to the solution $f_{1}$ is the operator $A_{2}=\frac{d^{2}}{d x^{2}}-\frac{6}{(x+1)^{2}}$ and by Theorem 19 the transmutation operator $\mathbf{T}_{2 ;-2}$ for $A_{2}$ is given by the Volterra integral operator (15) with the kernel

$$
\mathbf{K}_{2}(x, t ;-2)=-\frac{1}{(x+1)^{2}}\left(\int_{-t}^{x} \frac{-3 t+1}{2(s+1)}(s+1)^{2} d s+(1-t)^{2}\right)=\frac{(3 t-1)(x+1)^{2}-3(t-1)^{2}(t+1)}{4(x+1)^{2}} .
$$


This procedure may be continued iteratively. Consider operators

$$
A_{n}:=\frac{d^{2}}{d x^{2}}-\frac{n(n+1)}{(x+1)^{2}}
$$

$f_{n}(x)=(x+1)^{n+1}$ is a solution of the equation $A_{n} f=0$. The Darboux transformation of the operator $A_{n}$ with respect to the solution $f_{n}$ is the operator

$$
\frac{d^{2}}{d x^{2}}-2\left(\frac{f_{n}^{\prime}(x)}{f_{n}(x)}\right)^{2}+\frac{n(n+1)}{(x+1)^{2}}=\frac{d^{2}}{d x^{2}}-\frac{(n+1)(n+2)}{(x+1)^{2}},
$$

i.e., exactly the operator $A_{n+1}$. If we know $\mathbf{K}_{n}(x, t ;-n)$ for the operator $A_{n}$, by (18) we compute the kernel $\mathbf{K}_{n}(x, t ; n+1)$ corresponding to the solution $f_{n}(x)$ and by Theorem 19 we may calculate the kernel $\mathbf{K}_{n+1}(x, t ;-n-1)$. Careful analysis shows that we have to integrate only polynomials in all integrals involved, so the described procedure can be performed up to any fixed $n$.

Consider the Schrödinger equation

$$
u^{\prime \prime}+2 \operatorname{sech}^{2}(x) u=u .
$$

This equation appears in soliton theory and as an example of a reflectionless potential in the onedimensional quantum scattering theory (see, e.g. 22]). Equation (57) can be obtained as a result of the Darboux transformation of the equation $u^{\prime \prime}=u$ with respect to the solution $f(x)=\cosh x$. The transmutation operator for the operator $A_{1}=\partial_{x}^{2}-1$ was calculated in [3, Example 3]. Its kernel is given by the expression

$$
\mathbf{K}_{1}(x, t ; 0)=-\frac{1}{2} \frac{\sqrt{x^{2}-t^{2}} I_{1}\left(\sqrt{x^{2}-t^{2}}\right)}{x-t},
$$

where $I_{1}$ is the modified Bessel function of the first kind. Hence from Theorem 19 we obtain the transmutation kernel for the operator $A_{2}=\partial_{x}^{2}+2 \operatorname{sech}^{2} x-1$

$$
\mathbf{K}_{2}(x, t ; 0)=\frac{1}{2 \cosh (x)} \int_{-t}^{x}\left(\frac{I_{0}\left(\sqrt{s^{2}-t^{2}}\right) t}{s-t}+\frac{\sqrt{s^{2}-t^{2}} I_{1}\left(\sqrt{s^{2}-t^{2}}\right)}{(s-t)^{2}}\right) \cosh s d s .
$$

\section{Transmutation operator for the one-dimensional Dirac equa- tion with a Lorentz scalar potential}

One-dimensional Dirac equations with Lorentz scalar potentials are widely studied (see, for example, [5, 6, 12, 13, 14, 15, 16, 27, 32, 34] and [28, for intertwining techniques for it).

According to [27] the Dirac equation in one space dimension with a Lorentz scalar potential can be written as

$$
\begin{aligned}
\left(\partial_{x}+m+S(x)\right) \psi_{1} & =E \psi_{2}, \\
\left(-\partial_{x}+m+S(x)\right) \psi_{2} & =E \psi_{1},
\end{aligned}
$$

where $m(m>0)$ is the mass and $S(x)$ is a Lorentz scalar. Denote $\eta=m+S$ and write the system (58), (59) in a matrix form as

$$
\left(\begin{array}{cc}
\partial_{x}+\eta & 0 \\
0 & \partial_{x}-\eta
\end{array}\right)\left(\begin{array}{l}
\psi_{1} \\
\psi_{2}
\end{array}\right)=E\left(\begin{array}{cc}
0 & 1 \\
-1 & 0
\end{array}\right)\left(\begin{array}{l}
\psi_{1} \\
\psi_{2}
\end{array}\right)
$$

In order to apply the results on the transmutation operators and factorizations (42), (43) we consider a function $f$ such that

$$
\frac{f^{\prime}(x)}{f(x)}=-\eta=-m-S(x) .
$$


We can take $f(x)=\exp \left(-\int_{0}^{x}(m+S(s)) d s\right)$, then $f(0)=1$ and $f$ does not vanish. Suppose the operators $\mathbf{T}_{1}$ and $\mathbf{T}_{2}$ are transmutations for the operators $A_{1}=\left(\partial_{x}+\frac{f^{\prime}}{f}\right)\left(\partial_{x}-\frac{f^{\prime}}{f}\right)$ and $A_{2}=$ $\left(\partial_{x}-\frac{f^{\prime}}{f}\right)\left(\partial_{x}+\frac{f^{\prime}}{f}\right)$ respectively (corresponding to functions $f$ and $1 / f$ in the sense of Proposition 4 ). We will look for a solution of equation (60) in the form

$$
\left(\begin{array}{l}
\psi_{1} \\
\psi_{2}
\end{array}\right)=\left(\begin{array}{cc}
\mathbf{T}_{1} & 0 \\
0 & \mathbf{T}_{2}
\end{array}\right)\left(\begin{array}{l}
u_{1} \\
u_{2}
\end{array}\right)
$$

where $u_{1}$ and $u_{2}$ are some functions. From the commutation relations (53) and (54) we have

$$
\left(\begin{array}{cc}
\partial_{x}-\frac{f^{\prime}}{f} & 0 \\
0 & \partial_{x}+\frac{f^{\prime}}{f}
\end{array}\right)\left(\begin{array}{cc}
\mathbf{T}_{1} & 0 \\
0 & \mathbf{T}_{2}
\end{array}\right)\left(\begin{array}{l}
u_{1} \\
u_{2}
\end{array}\right)=\left(\begin{array}{cc}
f \partial_{x} \frac{1}{f} \mathbf{T}_{1} & 0 \\
0 & \frac{1}{f} \partial_{x} f \mathbf{T}_{2}
\end{array}\right)\left(\begin{array}{l}
u_{1} \\
u_{2}
\end{array}\right)=\left(\begin{array}{cc}
\mathbf{T}_{2} & 0 \\
0 & \mathbf{T}_{1}
\end{array}\right)\left(\begin{array}{cc}
\partial_{x} & 0 \\
0 & \partial_{x}
\end{array}\right)\left(\begin{array}{l}
u_{1} \\
u_{2}
\end{array}\right),
$$

hence

$$
\left(\begin{array}{cc}
\mathbf{T}_{2} & 0 \\
0 & \mathbf{T}_{1}
\end{array}\right)\left(\begin{array}{cc}
\partial_{x} & 0 \\
0 & \partial_{x}
\end{array}\right)\left(\begin{array}{l}
u_{1} \\
u_{2}
\end{array}\right)=E\left(\begin{array}{cc}
0 & 1 \\
-1 & 0
\end{array}\right)\left(\begin{array}{cc}
\mathbf{T}_{1} & 0 \\
0 & \mathbf{T}_{2}
\end{array}\right)\left(\begin{array}{l}
u_{1} \\
u_{2}
\end{array}\right)
$$

By multiplying both sides by the inverse matrix $\left(\begin{array}{cc}\mathbf{T}_{2}^{-1} & 0 \\ 0 & \mathbf{T}_{1}^{-1}\end{array}\right)$ we obtain

$$
\begin{aligned}
&\left(\begin{array}{cc}
\partial_{x} & 0 \\
0 & \partial_{x}
\end{array}\right)\left(\begin{array}{l}
u_{1} \\
u_{2}
\end{array}\right)=E\left(\begin{array}{cc}
\mathbf{T}_{2}^{-1} & 0 \\
0 & \mathbf{T}_{1}^{-1}
\end{array}\right)\left(\begin{array}{cc}
0 & 1 \\
-1 & 0
\end{array}\right)\left(\begin{array}{cc}
\mathbf{T}_{1} & 0 \\
0 & \mathbf{T}_{2}
\end{array}\right)\left(\begin{array}{l}
u_{1} \\
u_{2}
\end{array}\right)= \\
&=E\left(\begin{array}{cc}
0 & \mathbf{T}_{2}^{-1} \\
-\mathbf{T}_{1}^{-1} & 0
\end{array}\right)\left(\begin{array}{cc}
\mathbf{T}_{1} & 0 \\
0 & \mathbf{T}_{2}
\end{array}\right)\left(\begin{array}{l}
u_{1} \\
u_{2}
\end{array}\right)=E\left(\begin{array}{cc}
0 & 1 \\
-1 & 0
\end{array}\right)\left(\begin{array}{l}
u_{1} \\
u_{2}
\end{array}\right) .
\end{aligned}
$$

Therefore the operator $\left(\begin{array}{cc}\mathbf{T}_{1} & 0 \\ 0 & \mathbf{T}_{2}\end{array}\right)$ transmutes any solution $\left(\begin{array}{l}u_{1} \\ u_{2}\end{array}\right)$ of the system

$$
\begin{aligned}
& u_{1}^{\prime}=E u_{2} \\
& u_{2}^{\prime}=-E u_{1}
\end{aligned}
$$

into the solution $\left(\begin{array}{l}\psi_{1} \\ \psi_{2}\end{array}\right)$ of the system (58), (59) with the initial conditions $\psi_{1}(0)=u_{1}(0), \psi_{2}(0)=u_{2}(0)$. And vice versa if $\left(\begin{array}{l}\psi_{1} \\ \psi_{2}\end{array}\right)$ is a solution of the system (58), (59), then the operator $\left(\begin{array}{cc}\mathbf{T}_{1}^{-1} & 0 \\ 0 & \mathbf{T}_{2}^{-1}\end{array}\right)$ transmutes it into the solution $\left(\begin{array}{l}u_{1} \\ u_{2}\end{array}\right)$ of (61), (62) such that $u_{1}(0)=\psi_{1}(0), u_{2}(0)=\psi_{2}(0)$.

\section{Conclusions}

An explicit representation for the transmutation operator corresponding to a Darboux transformed Schrödinger operator is given in terms of the transmutation kernel for its superpartner and as a corollary the transmutation operator for the one-dimensional Dirac system with a scalar potential is obtained. Several examples of explicitly constructed transmutation operators are given. We expect that the techniques developed in the present paper will be used in practical applications of the transmutation operators.

\section{References}

[1] V. G. Bagrov and B. F. Samsonov. Darboux transformation, factorization, and supersymmetry in one-dimensional quantum mechanics, Teoret. Mat. Fiz. 1995, vol. 104, no. 2, 356-367 (in Russian); translation in Theoret. and Math. Phys. 1995, vol. 104, no. 2, 1051-1060.

[2] H. Begehr and R. Gilbert. Transformations, transmutations and kernel functions, vol. 1-2. Longman Scientific \& Technical, Harlow, 1992. 
[3] H. Campos, V. V. Kravchenko and S. Torba. Transmutations, L-bases and complete families of solutions of the stationary Schrödinger equation in the plane. Submitted to Journal of Mathematical Analysis and Applications. Available at arXiv:1109.5933

[4] R. W. Carroll. Transmutation theory and applications, Mathematics Studies, Vol. 117, North-Holland, 1985.

[5] J. Casahorrán. Solving smultaneously Dirac and Ricatti equations, Journal of Nonlinear Mathematical Physics, 1985, v.5, n.4, 371-382.

[6] C.-Y. Chen. Exact solutions of the Dirac equation with scalar and vector Hartmann potentials, Physics Letters A. 2005, vol. 339, 283-287.

[7] J. L. Cieśliński. Algebraic construction of the Darboux matrix revisited, J. Phys. A: Math. Theor. 2009, vol. 42, 404003.

[8] M. K. Fage and N. I. Nagnibida. The problem of equivalence of ordinary linear differential operators. Novosibirsk: Nauka, 1987 (in Russian).

[9] P. R. Garabedian. Partial differential equations. New York-London: John Willey and Sons, 1964.

[10] C. Gu, H. Hu, and Z. Zhou. Darboux Transformations in Integrable Systems, Springer-Verlag, Berlin, 2005.

[11] A. D. Hemery and A. P. Veselov. Whittaker-Hill equation and semifinite-gap Schrödinger operators, J. Math. Phys., 2010, Vol. 51, 072108; doi:10.1063/1.3455367.

[12] J. R. Hiller. Solution of the one-dimensional Dirac equation with a linear scalar potential, Am. J. Phys., 2002 , vol. $70(5), 522-524$.

[13] C.-L. Ho. Quasi-exact solvability of Dirac equation with Lorentz scalar potential, Ann. Physics 2006, vol. 321, no. 9, $2170-2182$.

[14] R. Jackiw and S.-Y. Pi. Persistence of zero modes in a gauged Dirac model for bilayer graphene, Phys. Rev. B, 2008, vol. 78, 132104.

[15] N. Kevlishvili, G. Piranishvili. Klein paradox in modified Dirac and Salpeter equations, Fizika, 2003, vol. 9, no. $3,4,57-61$.

[16] K. V. Khmelnytskaya and H. C. Rosu. An amplitude-phase (Ermakov-Lewis) approach for the Jackiw-Pi model of bilayer graphene, J. Phys. A: Math. Theor., 2009, vol. 42, 042004

[17] V. V. Kravchenko. A representation for solutions of the Sturm-Liouville equation. Complex Variables and Elliptic Equations, 2008, v. 53, 775-789.

[18] V. V. Kravchenko. Applied pseudoanalytic function theory. Basel: Birkhäuser, Series: Frontiers in Mathematics, 2009.

[19] V. V. Kravchenko. On the completeness of systems of recursive integrals. Communications in Mathematical Analysis, Conf. 032011 172-176.

[20] V. V. Kravchenko, S. Morelos and S. Tremblay. Complete systems of recursive integrals and Taylor series for solutions of Sturm-Liouville equations. To appear in Mathematical Methods in the Applied Sciences.

[21] V. V. Kravchenko and R. M. Porter. Spectral parameter power series for Sturm-Liouville problems. Mathematical Methods in the Applied Sciences 2010, v. 33, 459-468.

[22] G. L. Lamb. Elements of soliton theory. John Wiley \& Sons, New York, 1980.

[23] B. M. Levitan. Inverse Sturm-Liouville problems. VSP, Zeist, 1987.

[24] J. L. Lions. Solutions élémentaires de certains opérateurs différentiels à coefficients variables. Journ. de Math., t. 36, Fasc 1, 1957, 57-64.

[25] V. A. Marchenko. Sturm-Liouville operators and applications. Basel: Birkhäuser, 1986.

[26] V. Matveev and M. Salle. Darboux transformations and solitons. N.Y. Springer, 1991.

[27] Y. Nogami, F. M. Toyama. Supersymmetry aspects of the Dirac equation in one dimension with a Lorentz scalar potential, Physical Review A., 1993, vol. 47, no. 3, 1708-1714.

[28] L. M. Nieto, A. A. Pecheritsin, and B. F. Samsonov. Intertwining technique for the one-dimensional stationary Dirac equation, Annals of Physics 2003, vol. 305, 151-189.

[29] A. A. Pecheritsin, A. M. Pupasov and B. F. Samsonov. Singular matrix Darboux transformations in the inversescattering method, J. Phys. A: Math. Theor. 2011, vol. 44, 205305.

[30] C. Rogers, W. K. Schief. Backlund and Darboux transformations: geometry and modern applications in soliton theory, Cambridge University Press, 2002.

[31] H. Rosu. Short survey of Darboux transformations, Proceedings of "Symmetries in Quantum Mechanics and Quantum Optics", Burgos, Spain, 1999, 301-315.

[32] R. K. Roychoudhory, Y. P. Varshni. Shifted 1/N expansion and scalar potential in the Dirac equation, J. Phys. A: Math. Gen. 1987, vol. 20, L1083-L1087.

[33] S. M. Sitnik. Transmutations and applications: a survey. arXiv:1012.3741 1 [math.CA], originally published in the book: "Advances in Modern Analysis and Mathematical Modeling" Editors: Yu.F.Korobeinik, A.G.Kusraev, Vladikavkaz: Vladikavkaz Scientific Center of the Russian Academy of Sciences and Republic of North OssetiaAlania, 2008, 226-293. 
[34] R. Su, Yu Zhong and S. Hu. Solutions of Dirac equation with one-dimensional scalarlike potential, Chinese Phys.Lett., 1991, v.8, no.3, 114-117.

[35] K. Trimeche. Transmutation operators and mean-periodic functions associated with differential operators. London: Harwood Academic Publishers, 1988.

[36] V. S. Vladimirov. Equations of mathematical physics. Moskva: Nauka, 1981 (in Russian), English translation in V.S. Vladimirov. Equations of mathematical physics (2nd English ed.), Moscow: Mir Publishers, 1983. 